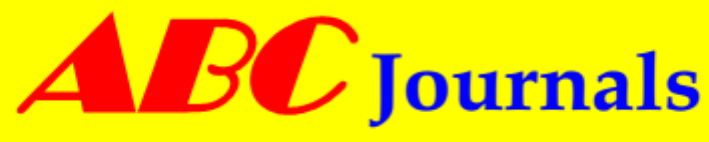

A new domain in research publishing

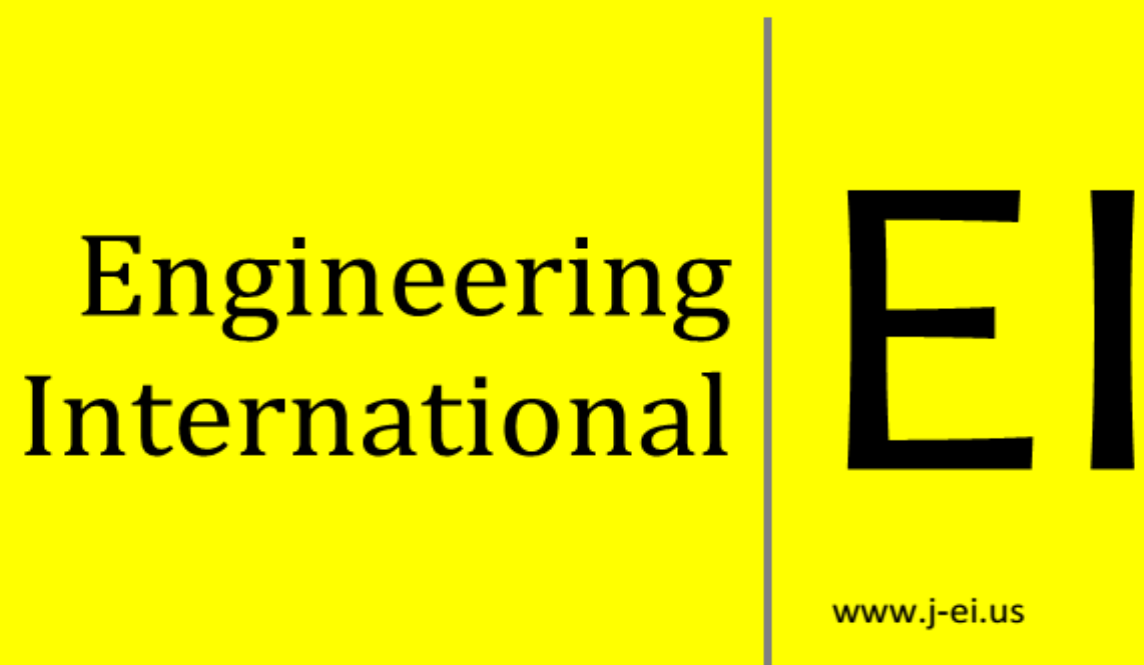

Asian Business Consortium 


\title{
Effects of Chemical Reaction and Heat Generation on MHD Boundary Layer Flow of a Moving Vertical Plate with Suction and Dissipation
}

\author{
M. Venkateswarlu'; G.V. Ramana $\operatorname{Reddy}^{2}$; \& D.V.Lakshmi ${ }^{3}$ \\ ${ }^{1}$ Department of Mathematics,V.R.Siddartha Engineering College, A.P., India \\ ${ }^{2}$ Department of Mathematics, K.L.University, A.P., India \\ ${ }^{3}$ Department of Mathematics, Bapatla Women Engineering College, A.P., India
}

\begin{abstract}
In this paper, the study of the steady two-dimensional flow of an incompressible viscous fluid with heat and mass transfer and MHD heat generation past a moving vertical plate with suction in the presence of viscous dissipation and chemical reaction is investigated. Using similarity variables, the governing partial differential equations are transformed into non-linear ordinary differential equations. These equations are then solved numerically using fourth order Runge-Kutta method with shooting technique. The flow variables are presented graphically. The graphs showed that velocity rises for increasing Grashof number, mass Grashof numer, suction, heat generation and Eckert number while reducing with increasing magnetic parameter, Schmidt number, and chemical reaction parameter and Prandtl number. Comparisons with previously published work are performed and are found to be in an excellent agreement.
\end{abstract}

Keywords: MHD, chemical reaction parameter, free convection, viscous dissipation, heat generation, suction, moving vertical plate.

\section{INTRODUCTION}

Convective flows with simultaneous heat and mass transfer under the influence of a magnetic field and chemical reaction arise in many transport processes both naturally and artificially in many branches of science and engineering applications. This phenomenon plays an important role in the chemical industry, power and cooling industry for dying, chemical vapour deposition on surfaces, cooling of nuclear reactors and petroleum industries. Natural convection flow occurs frequently in nature. It occurs due to temperature differences, as well as due to concentration differences or the combination of these two, for example in atmospheric flows, there exists differences in water concentration and hence the flow is influenced by such concentration difference. Changes in fluid density gradients may be caused by non-reversible chemical reaction in the system as well as by the differences in molecular weight between values of the reactants and the products. Chemical reaction can be modeled as either homogeneous or heterogeneous 
processes. This depends on whether they occur at an interface or as a single phase volume reaction. A homogeneous reaction is one that occurs uniformly throughout a given phase. On the other hand, a heterogeneous reaction takes place in a restricted area or within the boundary of the phase. In most cases of chemical reactions, the reaction rate depends on the concentration of the species itself. A reaction is said to be of first order, if the rate of reaction is directly proportional to the concentration itself, ( Cussler [1988]). For example, the formation of smog is a first order homogeneous reaction. Consider the emission of nitrogen dioxide from automobiles and other smoke-stacks. This nitrogen dioxide reacts chemically in the atmosphere with unburned hydrocarbons (aided by sunlight) and produces peroxyacetyl nitrate, which forms an envelope which is termed photo-chemical smog.

The study of heat and mass transfer with chemical reaction is of great practical importance in many branches of science and engineering. (Das et al [1994] ) studied the effects of mass transfer flow past an impulsively started infinite vertical plate with constant heat flux, and chemical reaction. (Anjalidevi and Kandasamy [1999] ) studied effects of chemical reaction, heat and mass transfer on laminar flow along a semi-infinite horizontal plate. More authors intensive studies have been carried out to investigate effects of chemical reaction on different flow types ( Seddeek et al [2007], Salem and Abd El- Aziz [2008], Mohamed [ 2009] Ibrahim et al [ 2008]).

Boundary layer behavior over a moving continuous solid surface is an important type of flow occurring in a number of engineering processes. To be more specific, heat treated materials traveling between a feed roll and a wind-up roll, aerodynamic extrusion of plastic sheets, glass fiber and paper production, cooling of an infinite metallic plate in a cooling path, manufacturing of polymeric sheets are examples for practical applications of continuous moving flat surfaces. Since the pioneering work of Sakiadis (1961) various aspects of the problem have been investigated by many authors. Mass transfer analysis at the stretched sheet were found in the studies by Erickson et al. (1966) and relevant experimental results were reported by Tsou et al. (1967) regarding several aspects for the flow and heat transfer boundary layer problems in a continuously moving sheet. Crane (1970) and Grubka (1985) have analyzed the stretching problem with constant surface temperature, while Soundalgekar (1974) investigated the Stokes problem for a viscoelastic fluid wall temperature and heat flux. Raptis and Singh (1985) studied flow past an impulsively started vertical plate in a porous medium by a finite difference method. The fluid considered in that paper is an optically dense viscous incompressible fluid of linearly varying temperature dependent viscosity. Ambethkar(2008) studied numerical solutions of heat and mass transfer effects of an unsteady MHD free convective flow past an infinite vertical plate with constant suction.

Alam and Rahman (2006) studied the combined free-forced convection and mass transfer flow past a vertical porous plate in a porous medium with heat generation and thermal diffusion. They also investigated MHD free convective heat and mass transfer flow past an inclined surface with heat generation. Salem (2006) discussed coupled heat and mass transfer in Darcy-Forchheimer mixed convection from a vertical flat plate embedded in a fluid saturated porous medium under the effects of radiation and viscous dissipation. Alam and Rahman (2008) analyzed the effects of chemical reaction and thermophoresis on MHD mixed convective heat and mass transfer flow along an inclined plate in the presence of heat generation/absorption with viscous dissipation and joule heating. Paresh Vyas and Ashutosh Ranjan (2010) discussed the dissipative MHD boundary- layer flow in a porous medium over a sheet stretching nonlinearly in the presence of radiation. Muthuraj and Srinivas(2009) studied the influence of magnetic field and wall slip 
conditions on steady flow between parallel flat wall and a long wavy wall with Soret effect. Ramana Reddy et.al (2012) studied Effects of the chemical reaction and radiation absorption on an unsteady mhd convective heat and mass transfer flow past a semiinfinite vertical moving in a porous medium with heat source and suction.

The study of heat generation or absorption in moving fluids is important in problems dealing with chemical reactions and those concerned with dissociating fluids. Heat generation effects may alter the temperature distribution and this in turn can affect the particle deposition rate in nuclear reactors, electronic chips and semi conductor wafers. Although exact modeling of internal heat generation or absorption is quite difficult, some simple mathematical models can be used to express its general behavior for most physical situations. Heat generation or absorption can be assumed to be constant, space-dependent or temperature-dependent. Tania et al (2010) has investigated the effects of radiation, heat generation and viscous dissipation on MHD free convection flow along a stretching sheet. Furthermore, Moalem (1976) studied the effect of temperature dependent heat sources taking place in electrically heating on the heat transfer within a porous medium. Vajravelu and Nayfeh (1992) reported on the hydro magnetic convection at a cone and a wedge in the presence of temperature dependent heat generation or absorption effects. Moreover, Chamkha (1999) studied the effect of heat generation or absorption on hydro magnetic three-dimensional free convection flow over a vertical stretching surface.

Viscous dissipation changes the temperature distributions by playing a role like an energy source, which leads to affected heat transfer rates. The merits of the effect of viscous dissipation depend on whether the plate is being cooled or heated. Heat transfer analysis over porous surface is of much practical interest due to its abundant applications. To be more specific, heat-treated materials traveling between a feed roll and wind-up roll or materials manufactured by extrusion. Glass-fiber and paper production, cooling of metallic sheets or electronic chips, crystal growing just to name a few. In these cases, the final product of desired characteristics depends on the rate of cooling in the process and the process of stretching. The work of Sonth et al (2002) deals with the effect of the viscous dissipation term along with temperature dependent heat source/sink on momentum, heat and mass transfer in a visco-elastic fluid flow over an accelerating surface. Chen (2004) examined the effect of combined heat and mass transfer on MHD free convection from a vertical surface with ohmic heating and viscous dissipation. The effect of viscous dissipation and joule heating on MHD free convection flow past a semi-infinite vertical flat plate in the presence of the combined effect of Hall and non-slip currents for the case of the power-law variation of the wall temperature is analyzed by Abo-Eldahab and El-Aziz (2005).Gupta et al (1977) studied heat and mass transfer on a stretching sheet with suction or blowing. Ibrahim and Makinde (2010) have investigated the effects of chemically reacting MHD boundary layer flow of heat and mass transfer over a moving vertical plate with suction.

This chapter aims to find numerical solutions of the coupled equations that govern the flow by using shooting technique with the forth order Range-Kutta method. In the problem formulation, the continuity, momentum, energy and concentration equations are reduced to some parameter problem by introducing suitable transformation variables. Pertinent results with respect to embedded parameters are displayed graphically for the velocity, temperature and concentration profiles and were discussed quantitatively. The local skin-friction coefficient and the heat and mass transfer results are obtained for representative values of the important parameters. 


\section{Mathematical Analysis}

Consider a two-dimensional free convective flow on the steady incompressible laminar MHD heat and mass transfer characteristics of a linearly started porous vertical plate, the velocity of the fluid far away from the plate surface is assumed zero for a quiescent state fluid. The flow configurations are linear. All the fluid properties are assumed to be constant except for the density variations in the buoyancy force term of the linear momentum equation. The magnetic Reynolds number is assumed to be small, so that the induced magnetic field is neglected. The Hall effects and the joule heating terms are also neglected. Then under Boussinesq's approximations, the governing boundary-layer equations that are based on the balance laws of mass, linear momentum, energy and concentration species for this investigation can be written as:

$\frac{\partial u}{\partial x}+\frac{\partial v}{\partial y}=0$

$u \frac{\partial u}{\partial x}+v \frac{\partial v}{\partial y}=v \frac{\partial^{2} u}{\partial y^{2}}-\frac{\sigma B_{0}^{2}}{\rho} u+g \beta_{T}\left(T-T_{\infty}\right)+g \beta_{C}\left(C-C_{\infty}\right)$

$u \frac{\partial T}{\partial x}+v \frac{\partial T}{\partial y}=\alpha \frac{\partial^{2} T}{\partial y^{2}}+\frac{Q_{0}}{\rho C_{p}}\left(T-T_{\infty}\right)+\frac{v}{C_{p}}\left(\frac{\partial u}{\partial y}\right)^{2}$

$u \frac{\partial C}{\partial x}+v \frac{\partial C}{\partial y}=D_{m} \frac{\partial^{2} C}{\partial y^{2}}-k r^{\prime}\left(C-C_{\infty}\right)$

The boundary conditions at the plate surface and for into the cold fluid may be written as $v=V, u=B x, T=T_{w}=T_{\infty}+a x, C=C_{w}=C_{\infty}+b x$, at $y=0$,

$u \rightarrow 0, T \rightarrow T_{\infty}, C \rightarrow C_{\infty}$ as $y \rightarrow \infty$

Where $B$ is constant, $a$ and $b$ denotes the stratification rate of the gradient of ambient temperature and concentration profiles. We introduce the following non-dimensional variables:

$$
\begin{aligned}
& \eta=y \sqrt{\frac{B}{v}}, \psi=x \sqrt{v B} f(\eta), \theta(\eta)=\frac{T-T_{\infty}}{T_{w}-T_{\infty}}, \phi(\eta)=\frac{C-C_{\infty}}{C_{w}-C_{\infty}}, M=\frac{\sigma B_{0}^{2}}{\rho B} \\
& E c=\frac{B^{2}}{C_{p}\left(T_{W}-T_{\infty}\right)}, G r=\frac{g \beta_{T}\left(T_{W}-T_{\infty}\right)}{x B^{2}}, G c=\frac{g \beta_{C}\left(C_{W}-C_{\infty}\right)}{x B^{2}}, \operatorname{Pr}=\frac{v}{\alpha}, \\
& S c=\frac{v}{D_{m}}, Q=\frac{Q_{0}}{\rho C_{p} B}, F=\frac{V}{\sqrt{B v}}, k r=\frac{k r^{\prime} B^{2}}{v}
\end{aligned}
$$

The velocity components $u$ and $v$ are respectively obtained as follows:

$$
u=\frac{\partial \psi}{\partial y}=x B f^{\prime}(\eta), \quad v=-\frac{\partial \psi}{\partial x}=-\sqrt{B v} f
$$

With this new set of independent and dependent variables defined by equation (6), the partial differential equations (2) to (4) are transformed into local similarity equations as follows:

$$
f^{\prime \prime \prime}+f f^{\prime \prime}-f^{\prime}\left(f^{\prime}+M\right)+G r \theta+G c \phi=0
$$




$$
\begin{aligned}
& \theta^{\prime \prime}+\operatorname{Pr} f \theta^{\prime}-\operatorname{Pr} f^{\prime} \theta+\operatorname{Pr} Q \theta+\operatorname{Pr} E c\left(f^{\prime \prime}\right)^{2}=0 \\
& \phi^{\prime \prime}+S c f \phi^{\prime}-k r S c \phi=0
\end{aligned}
$$

The corresponding boundary conditions (5) then take the following form

$$
\begin{aligned}
& f^{\prime}(0)=1, f(0)=-F w, \theta(0)=1, \phi(0)=1 \\
& f^{\prime}(\infty)=0, \theta(\infty)=0, \phi(\infty)=0
\end{aligned}
$$

where prime denotes partial differentiation with respect to $\eta$.

\section{Numerical Method of Solution}

The set of coupled non-linear governing boundary layer equations (8)-(10) together with the boundary conditions (11\&12) are solved numerically by using Runge-Kutta fourth order technique along with shooting method. First of all, higher order non-linear differential Equations (8)-(10) are converted into simultaneous linear differential equations of first order and they are further transformed into initial value problem by applying the shooting technique (Alam et al. (2006)). In a shooting method, the missing (unspecified) initial condition at the initial point of the interval is assumed, and the differential equation is then integrated numerically as an initial value problem to the terminal point. The accuracy of the assumed missing initial condition is then checked by comparing the calculated value of the dependent variable at the terminal point with its given value there. If a difference exists, another value of the missing initial condition must be assumed and the process is repeated. This process is continued until the agreement between the calculated and the given condition at the terminal point is within the specified degree of accuracy. For this type of iterative approach, one naturally inquires whether or not there is a systematic way of finding each succeeding (assumed) value of the missing initial condition. The resultant initial value problem is solved by employing Runge-Kutta fourth order technique. The step size $=0.05$ is used to obtain the numerical solution with decimal place accuracy as the criterion of convergence. The parameters of engineering interest for the present problem are the local skin friction coefficient, the local Nusselt number and the local Sherwood number, which are respectively proportional to $f^{\prime \prime}(0),-\theta^{\prime}(0)$, and $-\phi^{\prime}(0)$ are worked out and their numerical values presented in a tabular form.

\section{Comparison With Previous Work}

In the absence of heat generation and viscous dissipation, the results have been compared with that of Ibrahim and Makinde which are shown in Table 1. From this Table1, it can be clearly seen that the results are in good agreement with that of Ibrahim and Makinde (2010).

From Table 1 and Table 2. It is important to note that the local skin friction together with the local heat and mass transfer rate at the moving plate surface increases with increasing intensity of buoyancy forces $(G r, G c)$, the Schmidt number $(S c)$, the chemical reaction parameter. However, an increase in the magnetic field $(M)$, magnitude of fluid suction (Fw), heat source/sink parameter $(Q)$ and Viscous dissipation $(E c)$ causes a decrease in both skin friction and surface heat transfer rate and an increase in the surface mass transfer rate.

\section{Results and Discussion}

The governing equations (8)-(10) subject to the boundary conditions (11)-(12) are integrated as described in section 3. The Prandtl number was taken to be $\mathrm{Pr}=0.72$ which corresponds to air, 
the value of Schmidt number $(S c)$ were chosen to be $S_{c}=0.24,0.62,0.78,2.62$, representing diffusing chemical species of most common interest in air like $\mathrm{H}_{2}, \mathrm{H}_{2} \mathrm{O}, \mathrm{NH}_{3}$ and Propel Benzene respectively. Paying attention on positive value of the buoyancy parameters that is, local temperature Grashof number $\mathrm{Gr}>0$ and local concentration Grashof number $\mathrm{Gc}>0$. Throughout the calculations, the parametric values are fixed to be $G r=G c=E c=Q=M=0.1$, $S c=0.62, \operatorname{Pr}=0.72, K r=0.5$, and $F_{w}=0.1$, unless otherwise indicated.

The effects of various parameters on velocity profiles in the boundary layer are shown in Figs. 1-9. It is noticed from Figs. 1-9, that the velocity is higher near the moving vertical plate surface and decrease to its zero value far away from the moving vertical plate surface satisfying the far field boundary condition for all parameter values.

In Fig. 1 the effect of increasing the magnetic field strength on the momentum boundary layer thickness is illustrated. It is now a well established fact that the magnetic field presents a damping effect on the velocity field by creating drag force that opposes the fluid motion, causing the velocity to decease. However, in this case an increase in the $M$ only slightly slows down the motion of the fluid away from the moving vertical plate surface towards the free stream velocity, while the fluid velocity near the moving vertical plate surface decreases.

Figs. 2, 3, 4 \& 6 depict the variation of the boundary-layer velocity with the buoyancy forces parameters $(G r, G c)$, magnitude of fluid suction $(F w)$ and heat source/sink parameter $(Q)$. In both cases an upward acceleration of the fluid in the vicinity of the vertical wall is observed with increasing intensity of buoyancy forces. Further downstream of the fluid motion decelerates to the free stream velocity. Fig. 5 and Fig.9 shows that a slight decrease in the fluid velocity with an increase in the Schmidt number $(S c)$ and chemical reaction parameter. The effect of viscous dissipation parameter i.e., the Eckert number $E_{c}$ on the velocity component is shown in Fig. 7. The positive Eckert number implies cooling of the plate i.e., loss of heat from the plate to the fluid. Hence, greater viscous dissipative heat causes a slightly increase in the velocity. Fig. 8. Illustrates the velocity component for different values of the Prandtl number $\mathrm{Pr}$. The numerical results show that the effect of increasing values of Prandtl number results in a decreasing velocity.

In general the fluid temperature attains its maximum value at the moving vertical plate surface and decreases exponentially to the free stream zero value away from the plate satisfying the boundary condition. This is observed in Figs. 10-18. From these figures, it is interesting to note that the thermal boundary layer thickness decreases with an increase in the intensity of the buoyancy forces $(G r, G c)$ and Prandtl number $(P r)$. Moreover, the fluid temperature increases with an increase in the Schmidt number $(S c)$ the chemical reaction parameter $(k r)$, Magnetic field $(M)$, heat source/sink parameter $(Q)$ magnitude of fluid suction $(F w)$ and Eckert number (Ec) leading to an increase in thermal boundary layer thickness.

Figs. 19-27 illustrate chemical species concentration profiles against span wise coordinate $\eta$ for varying values physical parameters in the boundary layer. The species concentration is highest at the moving vertical plate surface and decrease to zero far away from the moving vertical plate satisfying the boundary condition. From these figures, it is important to reveal that the concentration boundary layer thickness decreases with an increase in, the buoyancy forces $(G r, G c)$, Schmidt number $(S c)$ the chemical reaction parameter $(k r)$, heat source/sink parameter $(Q)$ and Eckert number $(E c)$. Moreover, the fluid concentration increases with an increase in the magnetic field $(M)$ magnitude of fluid suction (Fw) and Prandtl number $(\mathrm{Pr})$ leading to an increase in thermal boundary layer thickness. 
Engineering International, Volume 1, No 1 (2013)

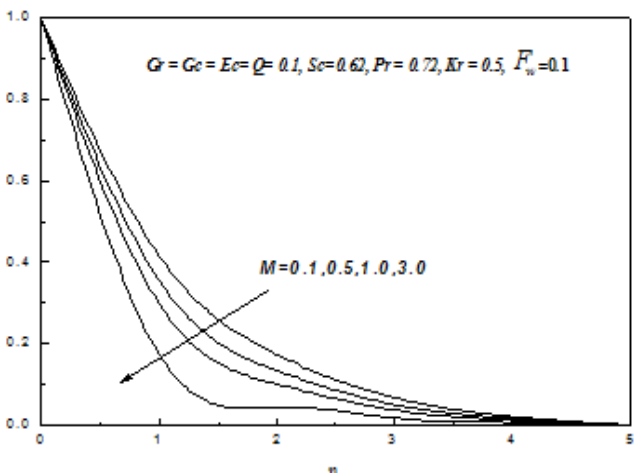

Fig. 1: Effect of the magnetic parameter $(M)$ on velocity profiles.

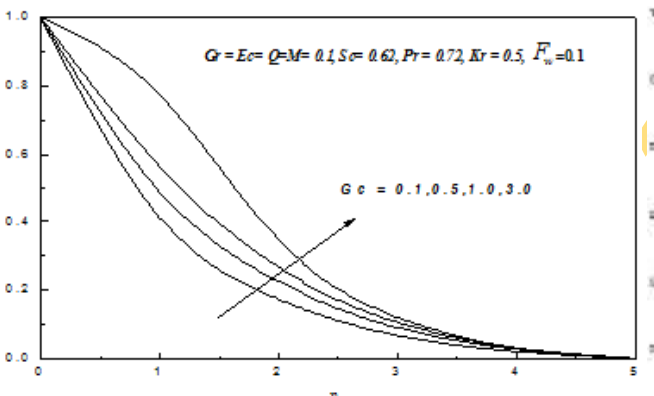

Fig. 3: Effect of the mass Grashof number $(\mathrm{Gc})$ on velocity profile

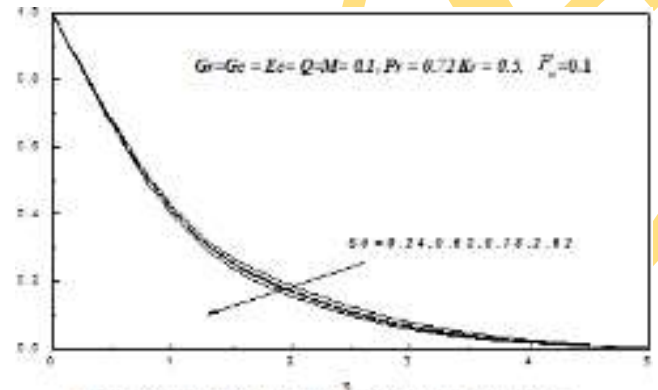

Fug. 5. Effost of the Schenidt number (3c) on velocity profiles

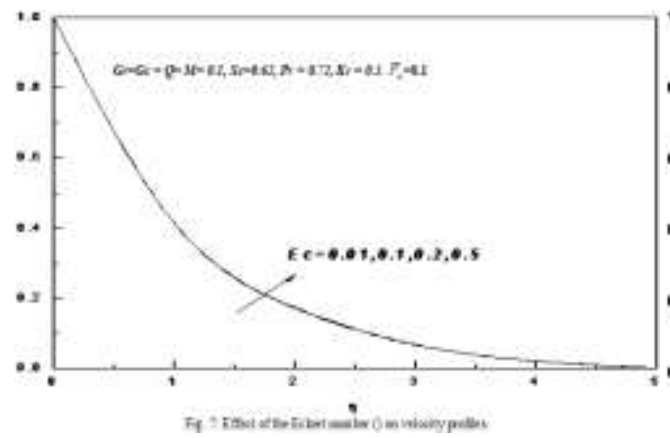

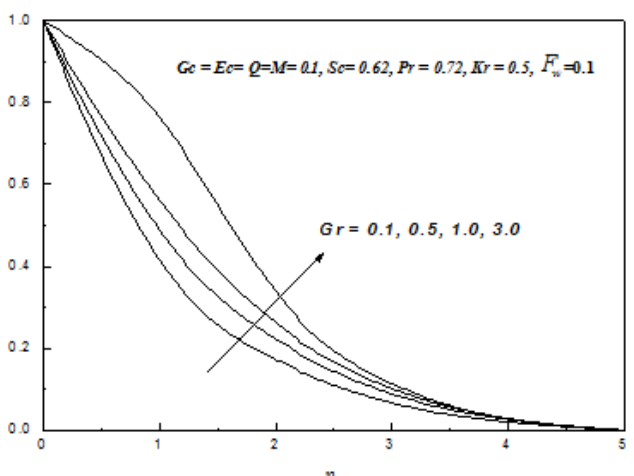

Fig. 2: Effect of the Grashof number (Gr) on velocity profiles

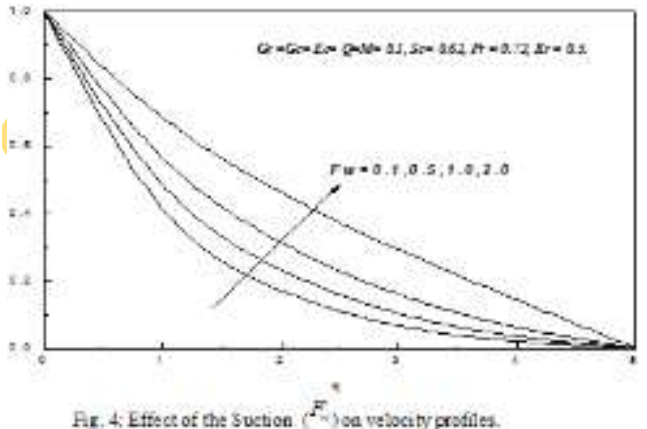

Ar. 4. Effect of the suction (F) oa velocity profiles.

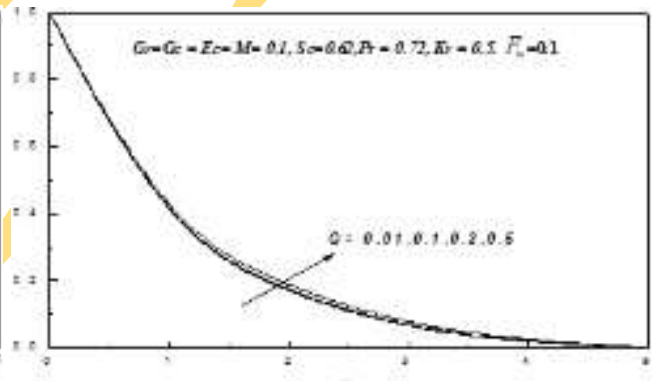

Fig. \&: Effoct of the teat genertisen peameter (Q) on velocity profiles

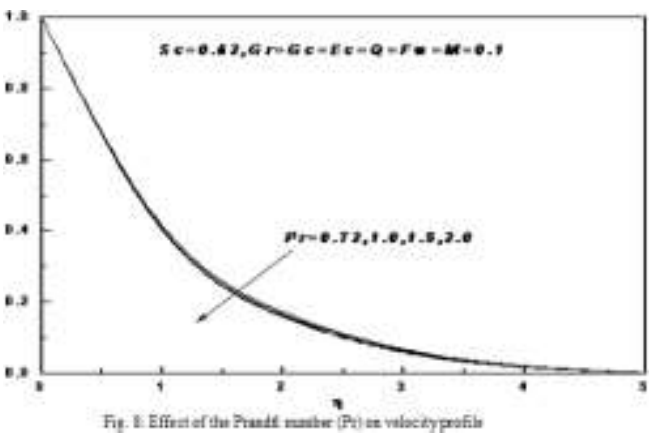


Engineering International, Volume 1, No 1 (2013)

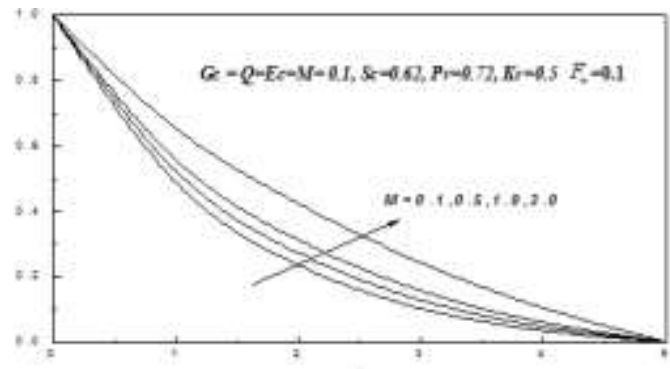

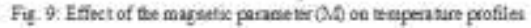

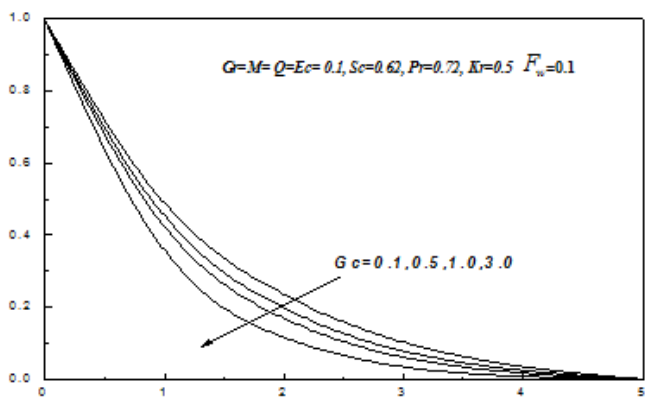

Fig. 11: Effect of the mass Grashof parameter $(\mathrm{Gc})$ on tempera ture profiles.

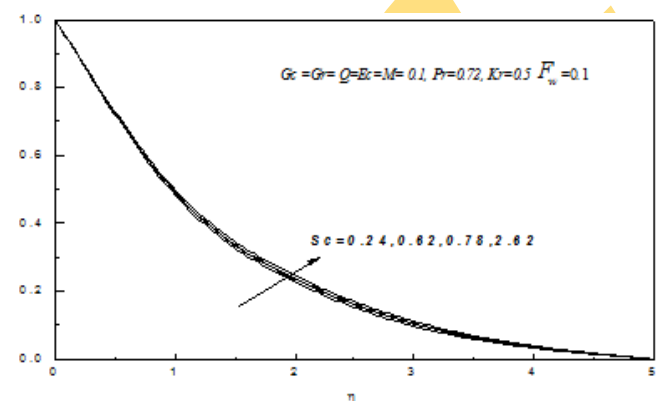

Fig. 13: Effect of the Schmidt number (Sc) on temperature profiles

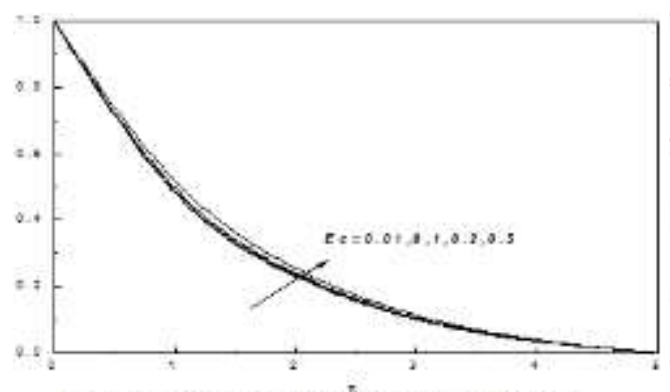

Fig. 15 - Efficet of the Fekert tumber (Fr) on tenpenaire profites

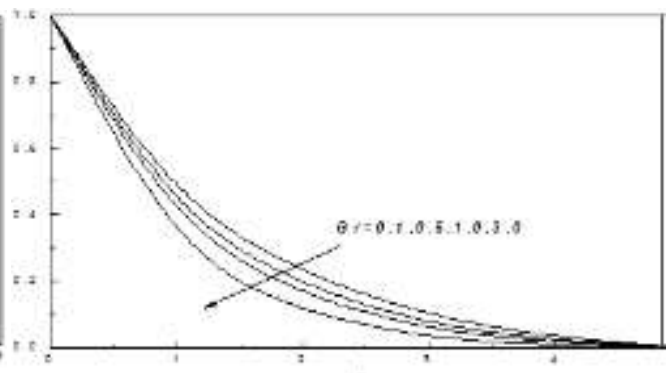

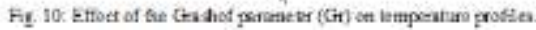

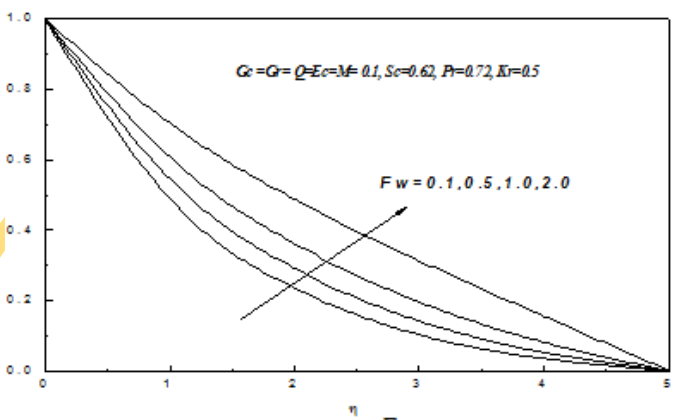

Fig. 12: Effect of the suction parameter $\left(F_{w}\right)$ on temperature profiles.

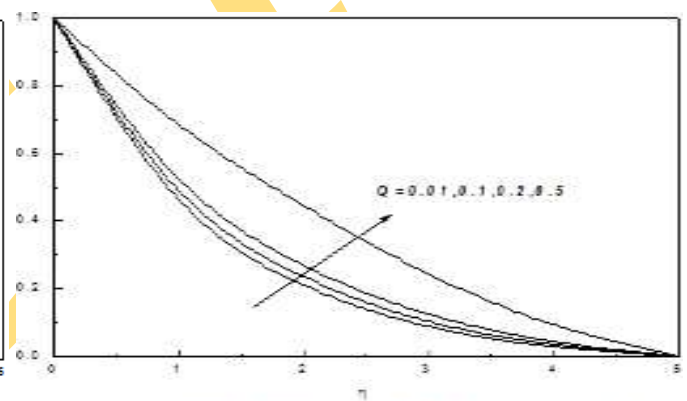

Fig. 14: Effect of the heat geteration parmeter $(Q)$ on temperatse profles:

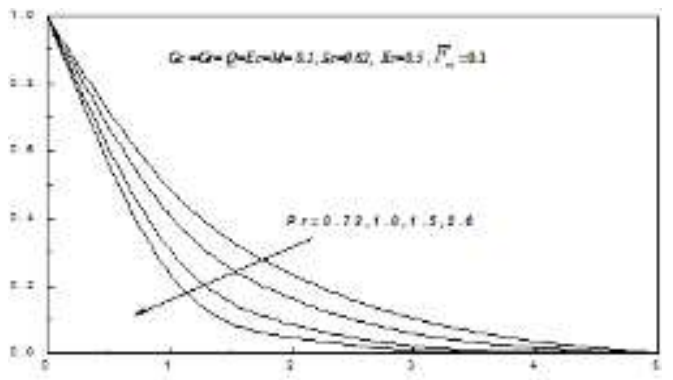

is. 15 - Effect of the Pratdit number (Pr) on tempraher profites 
Engineering International, Volume 1, No 1 (2013)
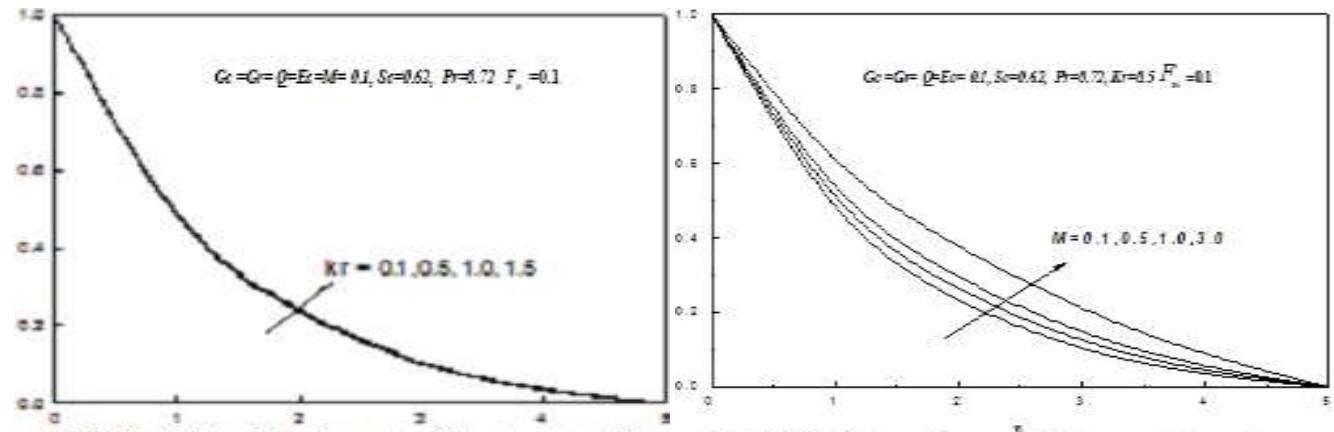

Fig. 17. Efect of the cierical reactisn pameter (Ki) on temperaure profiles

Fig. 18: Effect of the magretic parameter (M) ca concenra zon profiles.

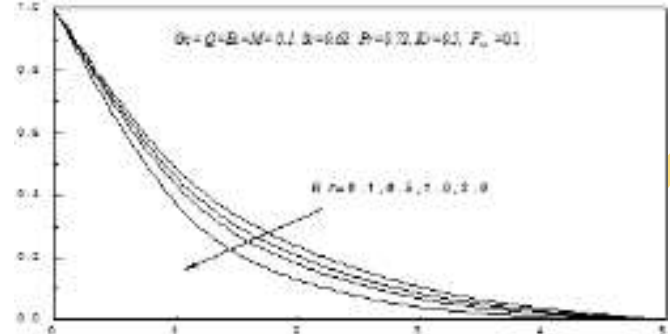

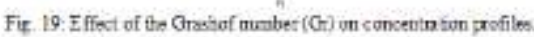

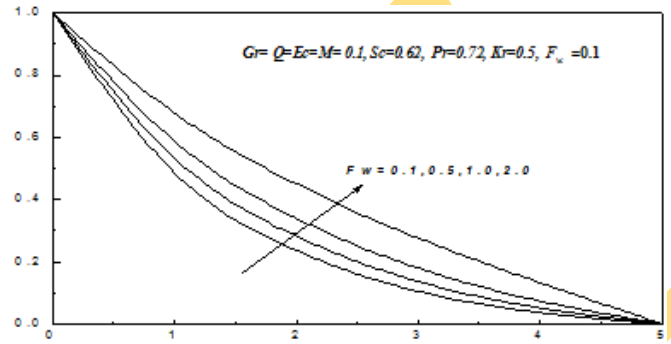

Fig. 21: Effect of the suction $\left(F^{n}\right)$ on concentration profiles.

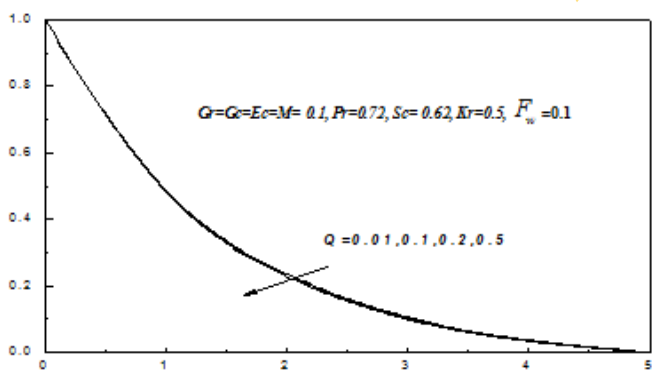

Fig. 23: Effect of the heat generation parameter $(Q)$ on concentration profiles

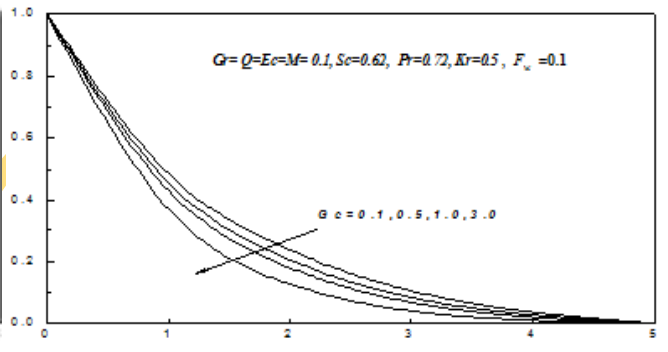

Fig. 20: Effect of the mass Grashof number $(\mathrm{Gc})$ on concentration profiles.

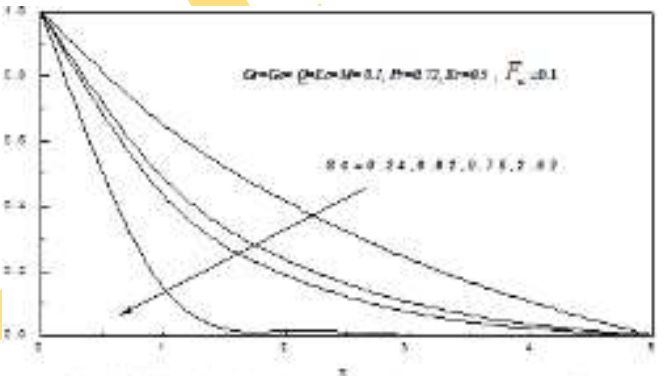

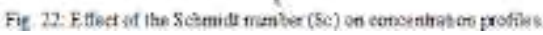

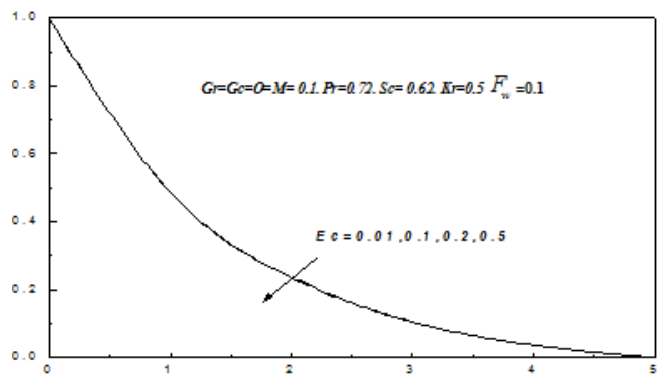

Fig. 24: Effect of the Eckert number $(\mathrm{Ec})$ on concentration profile 
Engineering International, Volume 1, No 1 (2013)

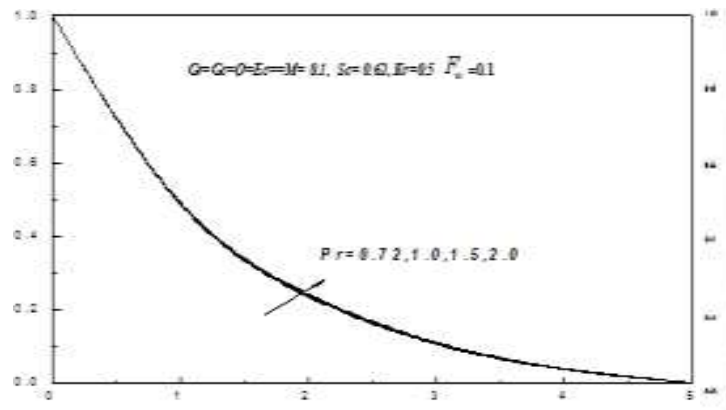

Fig. 25- Effact of the Prandt number (A) on osocentaicon profiles

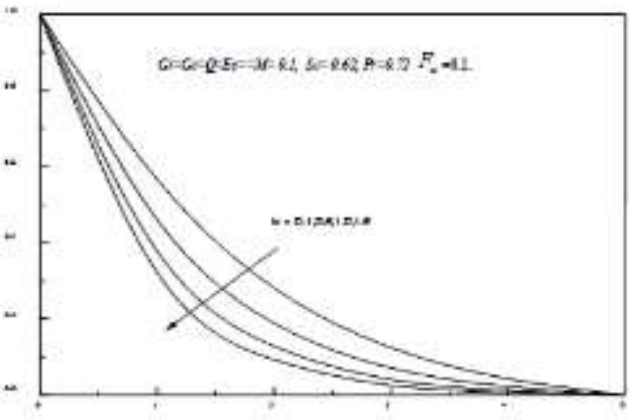

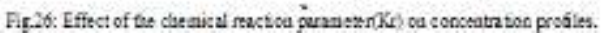

Table 1: variation of $f^{\prime \prime}(0), \theta^{\prime}(0)$ and $\phi^{\prime}(0)$ at the plate with $G r, G c, M, F w, S c$ for $\operatorname{Pr}=0.72$, $Q=E c=0$.

\begin{tabular}{|l|l|l|l|l|l|c|c|c|c|c|c|}
\hline$G r$ & $G c$ & $M$ & $F w$ & $S c$ & \multicolumn{2}{c|}{ Ibrahim and Makinde (2010) } & \multicolumn{3}{c|}{ Present work } \\
\hline \multicolumn{9}{|c|}{} & \multicolumn{9}{|c|}{} & $f^{\prime \prime}(0)$ & $-\theta^{\prime}(\mathbf{0})$ & $-\phi^{\prime}(\mathbf{0})$ & $f^{\prime \prime}(0)$ & $-\theta^{\prime}(\mathbf{0})$ & $-\phi^{\prime}(\mathbf{0})$ \\
\hline 0.1 & 0.1 & 0.1 & 0.1 & 0.62 & 0.888971 & 0.7965511 & 0.725392 & 0.889085 & 0.79653 & 0.725477 \\
\hline 0.5 & 0.1 & 0.1 & 0.1 & 0.62 & 0.695974 & 0.8379008 & 0.7658018 & 0.696036 & 0.837878 & 0.765851 \\
\hline 1.0 & 0.1 & 0.1 & 0.1 & 0.62 & 0.475058 & 0.8752835 & 0.8020042 & 0.475093 & 0.875269 & 0.802026 \\
\hline 0.1 & 0.5 & 0.1 & 0.1 & 0.62 & 0.686927 & 0.8421370 & 0.7701717 & 0.687021 & 0.842077 & 0.770165 \\
\hline 0.1 & 1.0 & 0.1 & 0.1 & 0.62 & 0.457723 & 0.8818619 & 0.8087332 & 0.457782 & 0.881824 & 0.808717 \\
\hline 0.1 & 0.1 & 1.0 & 0.1 & 0.62 & 1.264488 & 0.7089150 & 0.6400051 & 1.264045 & 0.708798 & 0.640369 \\
\hline 0.1 & 0.1 & 3.0 & 0.1 & 0.62 & 1.868158 & 0.5825119 & 0.5204793 & 1.867845 & 0.582456 & 1.866548 \\
\hline 0.1 & 0.1 & 0.1 & 1.0 & 0.62 & 0.570663 & 0.5601256 & 0.5271504 & 0.570745 & 0.56011 & 0.527309 \\
\hline 0.1 & 0.1 & 0.1 & 3.0 & 0.62 & 0.275153 & 0.2955702 & 0.2902427 & 0.276071 & 0.299108 & 0.296673 \\
\hline 0.1 & 0.1 & 0.1 & 0.1 & 0.78 & 0.893454 & 0.7936791 & 0.8339779 & 0.893518 & 0.79374 & 0.833984 \\
\hline 0.1 & 0.1 & 0.1 & 0.1 & 2.62 & 0.912307 & 0.7847840 & 1.6504511 & 0.91237 & 0.784892 & 1.65042 \\
\hline
\end{tabular}

Table 2: Variation of $f^{\prime \prime}(0), \theta^{\prime}(0)$ and $\phi^{\prime}(0)$ at the plate with $\mathrm{Q}$ and Ec. For $G r=G c=M=$ $F w=k r=0, S c=0.62, P r=0.72$.

\begin{tabular}{|l|l|l|l|l|l|}
\hline$Q$ & $E c$ & $k r$ & $f^{\prime \prime}(0)$ & $-\theta^{\prime}(0)$ & $-\phi^{\prime}(0)$ \\
\hline 0.1 & 0.1 & 0.5 & 0.89046 & 0.720703 & 0.732828 \\
\hline 0.5 & 0.1 & 0.5 & 0.876567 & 0.407081 & 0.739642 \\
\hline 1.0 & 0.1 & 0.5 & 0.860109 & 0.105458 & 0.74781 \\
\hline 0.1 & 0.5 & 0.5 & 0.888471 & 0.626001 & 0.733596 \\
\hline 0.1 & 1.0 & 0.5 & 0.886012 & 0.508875 & 0.734544 \\
\hline 0.1 & 0.1 & 1.0 & 0.894431 & 0.706313 & 0.886103 \\
\hline 0.1 & 0.1 & 1.5 & 0.898013 & 0.704035 & 1.04547 \\
\hline
\end{tabular}

\section{Conclusion}

In this paper the effect of heat generation and viscous dissipation on MHD boundary layer flow of a moving vertical flat plate with suction have been studied numerically. Shooting method along with fourth order Runge-Kutta algorithm is employed to integrate the equations governing the flow. Comparison with previously published work is performed and excellent argument has been observed. From the present numerical investigation, following conclusions may be drawn:

- The thermal and concentration boundary layer thickness decreases with an increase in the intensity of the buoyancy forces and. Gr Gc. 
- For increased value of magnetic parameter, the velocity profile decreases but the temperature and concentration profile increases slightly.

- The fluid temperature and concentration increases leading to an increase in thermal boundary layer thickness.

- An increase in wall suction increases the boundary layer thickness and decreases the skin friction.

- In general, the presence of the heat generation term in the energy equation yields an augment in the fluid's temperature

- The velocity, concentration distribution within the boundary layer decreases with the increase in values of the Schmidt number and the chemical reaction parameter.

- The velocity, temperature distribution within the boundary layer increases with the increase in values of the viscous dissipation.

\section{REFERENCES}

Abo-Eldahab E.M, El Aziz M.A (2005) Viscous dissipation and joule heating effects on MHD free convection from a vertical plate with power-law variation in surface temperature in the presence of Hall and iso-slips currents, Appl. Math. Model. Vol.29, pp.579-595.

Alam, M. S., Rahman, M. M. and Samad, M. A. (2006): Numerical study of the combined free-forced convection and mass transfer flow past a vertical porous plate in a porous medium with heat generation and thermal diffusion, Nonlinear Analysis; Modeling and Control, Vol. 11, No. 4, pp. 331-343

Alam, M. S., Rahman, M. M. and Sattar, M. A. (2006): MHD free convective heat and mass transfer flow past an inclined surface with heat generation, Thammasat Int. J. Sci. and Tech., Vol. 11, No. 4, pp. 1-8

Alam, M. S., Rahman, M. M. and Sattar, M. A. (2008): Effects of chemical reaction and thermophoresis on MHD mixed convective heat and mass transfer flow along an inclined plate in the presence of heat generation/absorption with viscous dissipation and joule heating, Canadian Journal of Physics, Vol. 86, pp. 1057-1066.

Ambethkar, V(2008): Numerical Solutions of Heat and Mass Transfer Effects of an Unsteady MHD Free Convective Flow past an Infinite Vertical Plate with Constant Suction, Journal of Naval Architecture and Marine Engineering, Vol. 5, No. 1 ,pp.27-36.

Anjalidevi S.P and Kandawamy R (1999). Effects of chemical reaction, heat and mass transfer on laminar flow along a semi-infinite horizontal plate. Heat and Mass Transfer, Vol. 35, pp. 465-467.

Chamkha A. J.(1999): Hydro magnetic three-dimensional free convection on a vertical stretching surface with heat generation or absorption, Int. J. Heat and Fluid Flow, 20, pp. 84-92 .

Chen C.H (2004) Combined heat and mass transfer in MHD free convection from a vertical surface with ohmic heating and viscous dissipation, Int. J. Eng. Sci., Vol.42, pp.699-713.

Crane, L.J. (1970): Flow past a Stretching Plate, Z. Angew. Math. Phys. Vol. 21, pp. 645-647.

Cussler E.L (1998). Difusion Mass Transfer in Fluid systems, Cambridge University Press, London.

Das U.N., Deka R and Soundalgekar V.M ( 1994). Effects of mass transfer on flow past an impulsively started infinite vertical plate with constant heat flux and chemical reaction. J. Forshung Im Ingenieurwesen- Engineering Research Bd, Vol. 60, pp. 284-287.

Erickson, L.E., Fan, L.T. and Fox, V. G. (1966): Heat and Mass Transfer on a Continuous Moving Surface, Ind. Eng. Chem. Fund. Vol. 5, No. 1, pp. 19-25.

Grubka, L. J. and Bobba, K. M. (1985): Heat Transfer Characteristics of a Continuous Stretching Surface with Variable Temperature, J Heat Transfer, 107, No. 1, pp. 248-250.

Gupta, P. S., Gupta, A. S. (1977): Heat and Mass Transfer on a Stretching Sheet with Suction or Blowing, Can. J. Chem. Eng, Vol. 55, No. 6, pp. 744-746.

Ibrahim and Makinde (2010): Chemically Reacting MHD Boundary Layer Flow of Heat and Mass Transfer over a Moving Vertical Plate with Suction, Scientific Research and Essays Vol. 5, No. 19 , pp. 2875-2882. 
Engineering International, Volume 1, No 1 (2013)

Ibrahim F.S., Elaiw A.M and Bakr AA (2008). Effect of chemical reaction and radiation absorption on the unsteady MHD free convection flow past a semi infinite vertical permeable moving plate with heat source and suction. Commun Nonlinear Sci. Numer. Simulate., Vol. 13, pp. 1056-1066.

Ingham, D. B and Pop, I.(Eds.) (1998): Transport Phenomena in Porous Media, Pergamon, Oxford.

Jain, M. K., Iyengar, S. R. K. and Jain, R. K. (1985): Numerical Methods for Scientific and Engineering Computation, Wiley Eastern Ltd., New Delhi, India.

Moalem .D.(1976) :Steady state heat transfer with porous medium with temperature dependent heat generation, Int. J. Heat and Mass Transfer, 19, pp. 529-537.

Mohamed R.A (2009). Double-Diffusive convection-radiation interaction on unsteady MHD flow over a vertical moving porous plate with heat generation and soret effects. Applied Mathematical Sciences, Vol. 3, No. 13, pp. 629-651.

Muthuraj.R and Srinivas.S (2009): Influence of magnetic field and wall slip conditions on steady flow between parallel flat wall and a long wavy wall with Soret effect. Journal of Naval Architecture and Marine Engineering, Vol 6, No 2 .

Na, T.Y. (1979): Computational Methods in Engineering Boundary Value Problems, Academic Press, New York.

Nield, D. A., and Bejan, A. (1998): Convection in Porous Media, 2nd. Ed., Springer-Verlag, Berlin.

Paresh Vyas and Ashutosh Ranjan (2010): Discussed the Dissipative MHD Boundary- Layer Flow in a Porous Medium over a Sheet Stretching Nonlinearly in the Presence of Radiation, Applied Mathematical Sciences, Vol. 4, No. 63, pp. 3133 - 3142

Raptis, A. and Singh, A. K. (1985): Free convection flow past an impulsively started vertical plate in a porous medium by finite difference method, Astrophysics. Space Sci., Vol. 112, pp. 259-265.

Sakiadis, B. C. (1961): Boundary-Layer Behavior on Continuous Solid Surfaces, Am. Inst. Chem. Eng. J., Vol. 7, No. 2, pp. 26-28.

Salem A.M and Abd El-Aziz M (2008). Effect of Hall currents and chemical reaction on hydromagnetic fos of a stretching vertical surface with internal heat generation/absorption. Applied Mathematical Modeling, Vol. 32, pp. 1236-1254.

Salem, A. M. (2006): Coupled Heat and Mass Transfer in Darcy-Forchheimer Mixed Convection from a Vertical Flat Plate Embedded in a Fluid Saturated Porous Medium under the Effects of Radiation and Viscous Dissipation, Journal of the Korean Physical Society, Vol. 48, No. 3, pp.109-113.

Seedeek M.A., Darwish A.A and Abdelmeguid M.S (2007). Effects of chemical reaction and variable viscosity on hydromagnetic mixed convection heat and mass transfer for Hiemenz flow through porous media with radiation, Commun Nonlinear Sci. Numer. Simulate., Vol. 15, pp. 195-213.

Seethamahalakshmi, G.V.Ramana Reddy and B. D. C. N Prasad (2012), Effects of the chemical reaction and radiation absorption on an unsteady $\mathrm{MHD}$ convective heat and mass transfer flow past a semi-infinite vertical moving in a porous medium with heat source and suction, IOSR Journal of Engineering (IOSRJEN) Vol. 1, Issue 1, pp. 028-036.

Sonth R.M, Khan S.K, Abel M.S and Prasad K.V (2002) Heat and mass transfer in a visco-elastic fluid over an accelerating surface with heat source/sink and viscous dissipation, Heat Mass Transfer, Vol. 38, pp.213-220.Soundalgekar, V. M. (1974): Stokes Problem for Elastic-Viscous Fluid, Rheol. Acta, Vol. 13, No. 2, pp. 177-179.

Tania, S. K. and Samad, M.A. (2010) Effects of radiation, heat generation and viscous dissipation on MHD free convection flow along a stretching sheet. Research J of Appl Sci, Eng and Tech 2(4), pp. 368-377.

Tsou, F. K., Sparrow, E. M. and Goldstein, R. J. (1967): Flow and Heat Transfer in the Boundary Layer on a Continuous Moving Surface, Int. J. Heat Mass Transfer, Vol. 10, pp. 219-235.

Vajrevelu. K., J. Nayfeh (1992): Hydro magnetic convection at a cone and a wedge, Int. Comm. Heat Mass Transfer, 19, pp. 701-710. 